\title{
LV. An account of test objects for microscopes
}

\section{Andrew Pritchard Esq.}

To cite this article: Andrew Pritchard Esq. (1833) LV. An account of test objects for microscopes, Philosophical Magazine Series 3, 2:11, 335-344, DOI: 10.1080/14786443308648053

To link to this article: http://dx.doi.org/10.1080/14786443308648053

曲 Published online: 01 Jun 2009.

Submit your article to this journal $₫$

Џ Article views: 2

Q View related articles $\sqsubset$ 
LV. An Account of Test Objects for Microscopes. By ANDrew Pritchard, Esq.*.

\author{
[With a Plate.]
}

FVERY important advance in our knowledge of those bodies in the material universe, from which our earth appears as an atom, has been coeval with, and greatly dependent upon, some augnentation of the powers and effectiveness of telescopes. Before the discovery of the double stars and nebulæ, the goodness of these instruments was determined by their capability of showing the planets and their satellites. But, since our acquaintance with the former bodies, telescopes have to undergo more severe tests, and greater accuracy in their construction is required. What has been advanced in regard to the telescope will be found applicable to the microscope; and to the discovery of certain objects which may be considered as tests of the penetrating and defining powers of this instrument, we may justly attribute the grand and magnificent improvements which the microscope has recently received.

In the perusal of the works of Leeuwenhoek, Dr. Goring met with a passage describing the dust, or imbricated scales, which cover the wings of the silkworm (Phalena Mori), from which he was led to suspect there were some peculiar properties in the lines on the feathers and scales of insects, rendering them more difficult to be discerned than other microscopic objects; and the result of his investigation was the discovery of their properties as tests-a description of object before unknown in the annals of microscopic science.

Now, as it is undoubtedly of the highest importance to the naturalist that he should know the exact capabilities of his instrument, in order that he may not be led astray in his investigations, by placing undue confidence in it; and as these tests offer the best means of accomplishing this end, I conceive them to be of the greatest value and interest. As no complete account of them is extant, I shall endeavour to supply this deficiency in the present chapter, and illustrate the subject by accurate drawings of them, greatly magnified. ***

Having ascertained that different test-objects require different degrees of perfection in the instrument used to develope their structure, it became an interesting pursuit to discover those which are best adapted for this purpose, and the peculiarities, in the illumination, \&c. under which they are exhi-

"Abridged from the "Microscopic Cabinet." London: Whittaker, Treacher and Co. 1832. An account of this work was given in the Lond. and Edinb. Phil. Mag, and Journ. vol. i. p. 163. 
bited with the greatest perspicuity. In this investigation, it was found that there were two distinct properties in a microscope, and that the instrument might possess a very considerable approximation to perfection in the one, and fall short in the other, or vice versâ, or might be perfect in both. The lines on the dust or feathers from the wings of the lepidoptera, and those on the scales from the body and limbs of the thysanuræous insects, offered the means of determining their goodness in one particular, viz. their penetration, and the structure of the hair of animals, certain mosses, \&c. served to ascertain their defining power.

The analogy between telescopes and microscopes is so great, that I cannot be said to digress from my subject by stating that the aforesaid observations apply also to the former of these instruments, which seldom combines the two qualities of penetration and definition to any great extent. Thus, a telescope with a large aperture will frequently resolve clusters of stars, and exhibit nebulæ, while it will fail in defining the disc of a planet, or the moon, with precision; and, on the other hand, one of moderate aperture accurately figured will define the latter, but be wholly inert on the nebula and clusters. So a microscope with large aperture and high power will show the " active molecules" and lined objects, while it will not define a leaf of moss, or a mouse hair; and another with a smaller aperture will define the latter, but prove ineffective on the former. This is very manifest in single lenses which require different apertures for different objects*.

The penetration of a microscope has been shown to be dependent on its angle of aperture, and that whenever this was less than a certain quantity, the lined structure of the scales cannot be rendered visible, however perfect the instrument may be; and the defining power is inversely as the quantity of spherical and chromatic aberration.

A proof, or test-object, may be defined to be one which requires a certain degree of excellence or perfection in a microscope or engiscope for the development either of the whole, or some particular part of its structure.

Test-objects are separable into two great divisions; but as I intend only to treat on one of them, it is proper here to point out their distinction. In the first division I place those which operate out of focus, and tell us what the defects of an instrument are. The second, those which, if exhibited by a micro-

* I have a very beautiful sapphire lens (plano-convex of one fifteenth focus) that shows the lines on the long brassica very distinct and sharp, when its aperture is large, but will not define a moss satisfactorily with this aperture; but as stops behind the object have the effect of reducing it, with them it shows the latter. 
scope, assure us that it possesses certain good qualities. The first division, as artificial stars, enamel dial-plate, wire gauze, \&c.*, which inform us of the state of their aberration, achromatism, centering, adjustment, curves, \&c., I shali pass over,as many persons are not disposed to enter into a scientific scrutiny concerning the causes of their denerits, and because they are more applicable to engiscopes, or compound microscopes, than to single and compound magnifiers, - -and shall content myself by giving some simple means of determining effectiveness by means of the second division.

(1.) Lepisma saccharina.-The insects of the families Lepismenæ and Podurellæ are comprehended in the order Thysanoura of Cuvier and Latreille; they are small, frequenting damp places, and are of various colours; they leap like fleas.

The scales of these apterous insects must be taken from fresh specimens, for, when long dead, they adhere so firmly to the insect, that they cannot be detached without injury.

Their longitudinal lines slightly radiate from the point of insertion; they are readily seen, and appear flat or square, like the indentations on some bivalve shells: these are the prettiest scales I am acquainted with. There are other lines in various directions, as shown in the drawing of a magnified scale at fig. I. Plate III. When the candle is so placed as to bring out the latter strongest, and the scale is turned round in the axis of the microscope in certain positions, they will cease to appear connected. In this object it is the sharpness and cleanness of the spaces that chiefly evince the goodness of a microscope, for the longitudinal lines are easily developed.

(2.) The Morpho Menelaus. - This butterfly is indigenous to America, the wings are indented, and their superior surface of a highly-polished blue colour.

The imbricated scales from the centre of the superior side of the wing are of a pale blue, mixt with others almost black. The former are mostly broader than the latter, and are the testobjects required; they measure about one one-hundred and twentieth of an inch in length. When viewed in a microscope, they exhibit a series of longitudinal stripes or lines, as shown in the magnified drawing, fig. 2. Plate III. Between these lines are disposed cross striæ, which, with the lines, give it the appearance of brick-work.

The microscope or engiscope under examination should be able to make out these markings, with the spaces between them, clean and distinct. The cross striæ, which give the

* For a particular account of these objects, see Dr. Goring”s Memoirs "On the Exact Method of, \&c." p. 191. Mic. Cab.

Third Series. Vol. 2. No. 11. May 1833. $2 \mathrm{X}$ 
brick-work appearance, are seldom to be seen all over the feather at once. The tissue that covers this scale or feather contains the largest portion of colouring matter, and is often destroyed in removing them from the wing, and along with it the cross striæ. In such cases, the longitudinal lines only can be visible. The damaged specimens are easily known by their paleness.

(3.) (Alucita pentadactylus, and hexadactylus.)-The ten and twenty Plumed Moths.-The structure of the wings, or, more properly, plumes of these insects, is so peculiar, that few persons acquainted with entomology are strangers to it.

The twenty-plumed moth is more delicate in its form than the other. The feathers or scales, employed as proof objects, must be taken from the body of the insect, and not from the plumes or wings. Their breadth is generally greater than their length, and their form is never symmetrical. They are transparent, and about one one-hundred and eightieth of an inch long. The scale is often partially covered by a delicate, uneven, membranous film, which obliterates the lines on those parts. The longitudinal lines are not difficult to resolve, but their proximity is such, that they require a considerable power and careful illumination to separate them distinctly. They are elegant microscopic objects, but rather scarce.

(5.) The Clothes Moth.-(Tinea vestianella.)-These small brown moths possess very delicate and unique scales, requiring some tact in the management of the illumination, to resolve their lines distinctly. I should observe, that it is the small feathers only, from the under side of the wing, that must be considered as tests; the others are easy. A magnified view of a small one, about one four-hundredth of an inch long, is given in fig. 3. of Plate III. They are readily made out under the single and doublet magnifiers. This is a favourite object with some, who exhibit it as the standard of excellence. I do not consider it very difficult; though it must be almitted, to bring out the lines sharp and clean, requires an excellent instrument.

(6.) Pontia Brassica* (Leach.)-The pale slender doubleheaded feathers, about one eightieth of an inch long, having brush-like appendages at their insertion, obtained from some portions of the wing of this large cabbage butterfly, afford an excellent criterion of the goodness of a microscope. Some connoisseurs prefer them to all others, and form an accurate judgement of an instrument by the manner in which it demonstrates this single object. They are easily detached from the

* This is the Pieris Brassica of Latreille. 
wing by the point of a quill, but must be gently handled, for, like many others, they are soon mutilated; indeed I have seldom seen them perfect in the ordinary sliders. Those specimens which are easily resolved are readily distinguished, being short, broad, and more opake. There are also found, on the same wing, two or three other sorts, but they are unworthy of notice as proof objects.

In Plate III. at fig. $4^{*}$. is represented a sample of the regular proof feather. It is very transparent, and has a yellowish tint; the surface is seldom smooth, as indicated at the part $a$. In the engiscope these inequalities are not so observable, and therefore, when the lines appear strong, the surface is more uniform than in the microscope. This object requires the light more oblique than any other of the lined kind. On this account I have seldom been able to see the lines satisfactorily with Dr. Wollaston's illumination, unless the magnifier was much out of the axis of the perforation. If we throw the light of a candle (placed a few inches behind the stage) obliquely on then, they can be seen very sharp. I have seen them in this way with a simple jewel lens, of only one fifteenth of an inch focus.

(7.) The Podura plumbea.-(Lead-coloured Spring-tail.)The body and limbs of these insects are covered with scales, which, from their extreme delicacy, require great care in removing. They are also very soft, and easily wounded. The fluid which exudes from the injury so completely adheres to the scales as to obliterate all their markings. Hence they must be cautiously handled. Those who are desirous of preserving these insects, should keep camphor along with them; through omitting this, I once had a large collection of them consumed by a species of mite (Acarus), which had insinuated itself into the box.

I have never been able to see the lines on them with a power much below 250 (that is, one twenty-fifth of an inch focus), and therefore microscopes of a lower power cannot be expected to show them, except of very superior quality ; for it must constantly be kept in mind, that that instrument is the best which exhibits an object with the least amplification, all other things being equal.

It is affirmed, by a very acute experimenter, of these scales, that " all are difficult, and some seem to defy all power of definition." The latter part of this quotation is perfectly accurate; but $I$ differ in the former, because many specimens,

* The reader should examine this and the other figures with a band magnifier. 
340 Mr. Pritchard's Account of Test Objects for Microscopes.

especially the French ones, are very easy, and unworthy the title of proofs; and, as they might be substituted for those $I$ am describing, and thus a common instrunient might pass for one of superior excellence, $I$ feel justified in giving this caution *.

The size of these scales varies from one nine hundredth to one hundred and sixtieth of an inch in length, and, as they decrease in size, become more transparent. They are of different forms, but possess a general character, easily recognised, by the want of any sharp angles. Under a microscope not having sufficient penetration, the tissue appears devoid of structure or markings; but, when placed in a superior one, and the illumination properly nade, they show a series of lines or cords on their surface, and present a much greater variety in their arrangement than the scales of any other species of insect. Some have the lines straight, as shown in the magnified scales, Plate III. fig. 5. and 6, and have two sets of oblique lines on them, similar to fig. $8+$; others are waved and curved, as shown in fig. 7,9 , and 10 , while on some of the small ones, as fig. 11, nothing satisfactory has yet been developed. In these figures I have endeavoured to give the appearances which the objects present under the microscope; and it will be observed, on a careful inspection of them, that the lines on fig. 9,10 , and 13, (which are only portions of scales, ) are very different from those on fig. 5 . and 6 , the former ones not being so sharp and defined as the latter.

As a general rule, it will be found that the smaller the scales the more difficult the test; those in fig. 6, however, cannot be included as tests, as they are very easily resolved. I must not omit to notice, also, that the cords on these scales are loosely attached to the tissue, and are often rubbed off in mounting. Of course it will be fruitless to examine such specimens. Those on which the greatest reliance may be placed are similar to fig. 5 , though the same scale will assume all the appearances of fig. $8,9,10$, and 13 .

Before leaving the subject of the lined objects, I should notice, that all objects of similar structure are more or less tests, as the lines on the scales of some beetles, one of which, from

* It should be remembered that the exhibition of the lines on these scales is only proof of the penetration of a microscope; and unless the outline of the scale is sharp. the instrument is defective in definition.

$\uparrow$ As in the scales of the Pontia Brassica, only one system of oblique lines can be seen at once; the other system is similar to those in fig. 8 , but running in a direction at right angles to them. 
the diamond beetle (Curculio imperialis) is shown at fig. 12 . Plate III.* The lines and markings on certain vegetable tissues, and many others too numerous to name, may also be employed as proof objects. The reason for making a selection of those above described, has been in order to render the task of judging of the merits of an instrument by clifferent individuals more simple and satisfactory, so that by the assistance of the drawings, and a sample of the objects, they may ascertain the quality of an instrument without the trouble of comparing it with oihers, which are often difficult, and sometimes impossible, to procure.

\section{Definition.}

The defining power of microscopes and engiscopes depends on their capability of collecting together all the rays from any one point of the object, or, in other words, their freedom from aberration, and is independent of their penetration; for, if we take an engiscope and view a lined object with the aperture of the objective, as it is usually sold in the shops, its defining power may be very fair; but if we enlarge the aperture so as to enable us to develope the lines which it will then accomplish, the defining power of the instrument will be injured to such an extent as to render the outline quite confused. The great desideratum, then, in microscopes and engiscopes, is to obtain these two qualities combined, which, however, is only rarely attained.

Cylindrical or spherical bodies appear the best suited for ascertaining the goodness of an instrument, as regards definition; and the following examples, which are prefaced by remarks on the method of illuminating them, I deem sufficient for this end.

In the preceding class of objects, oblique diverging rays ap-

* The scales from the body of the diamond beetle, either as transparent or opake objects, are by far the mest brilliant, in point of colour, of any of the lined class. In viewing them as opake objects, with single lenses, in order to exhibit the lines, the scale must be brought a little within the focus, and the illumination carefully arranged. As you cannot exhibit them with single lenses of a one twentieth or one thirtieth of an inch focus without using silver cups, it is difficult to procure oblique light. As transparent objects, they are much easier managed. 'They present a mottled sort of colour, composed of the brightest carmine, mixed with purple, blue, and yellow, and their lines are distinctly seen, as shown in fig. 1\%. As the lines on some of these scales are of easy resolution, it will not be advisable to trust every specimen as a test. The small ones from the legs of the Brazilian beetle are the most difficult, and many of these require the most rigid adjustment of the focus and illumination to resolve the lines, and the slightest tremor, though not enough to occasion any sensible dancing (as a carriage at a distance), is sufficient to render them invisible. 
342 Mr. Pritchard's Account of Test Objects for Microscopes.

pear to be essential for the development of their structure, the degree of obliquity varying, however, with different specimens of scales. The extremes of this variation are the Podura plumbea and Pieris Brassica, the delicacy of the former requiring almost central light, while the latter requires it very oblique. From this cause artificial illumination is to be preferred to day-light for this class of objects, as the light of a lamp or candle gives the rays diverging without any apparatus whatever. The same effect, however, may be produced in day-light, either with Dr. Wollaston's or Dr. Goring's illuminator, where the rays, after meeting at the focus of theiv illuminating lens, are permitted to diverge, and, by placing the object out of the centre, oblique vision is obtained. In the investigation of the class of objects now to be described, direct parallel rays are preferable, and, indeed, in most cases are essential; and on this account they are scarcely ever well defined by candle- or lamp-light. In these, therefore, clear day-light, directed through the axis of the instrument, should be employed.

1. The hairs of the common mouse (Mus domesticus) differ both in size and form; the principal varieties, with their relative diameters, are shown in Plate III. fig. 14, 15, and 16. 'These are drawn, as seen by transmitted light, and as proot:objects should have their transparent parts clearly and distinctly separated from the darker portions. This renark holds good for the whole tribe of hairs and mosses, and it is from the sharpness with which the parts are separated that a correct opinion of the goodness of an instrument can be obtained. When these hairs are seen by reflected light, that is, as opake objects, their appearance is altered, the dark solid parts reflecting more light than the transparent portion; hence they are lighter than the latter. A peculiar and interesting variety of a large hair viewed in this way is shown at fig. 17; it is engraved from a drawing made by Dr. Goring. ** *

3. The hair from the wing of the bat (Vespertilio murinus). -Although this creature is supposed to bear some affinity to that of the mouse, the structure of the hair of these two animals is entirely different: there are, however, great varieties, the principal of which are shown at fig. 18. and 19. The hair in the latter figure is spiral; the former like a succession of cones, the apex of one being inserted into the base of the following.

Many other kinds of hair might be enumerated for the purpose to which I have applied the above; but I deem these amply sufficient to illustrate this part of the subject. As, however, the diversities in the structure of different kinds of hair 
are worthy of investigation*, I have sketched a few of the most interesting varieties. They are all magnified in the same proportion as the mouse and bat's hairs, which accompany them in Plate III.

Fig. 20. is a hair from the larva of the common dermestes.

Fig. 21. is a white hair from a young cat.

Fig. 22. is the hair of a Siberian fox; and

Fig. 23. the hair of a common caterpillart.

4. The Lycana Argus. - Among the scales on the underside of the wing of this elegant blue butterfly, are some whose conformation is remarkably singular; their form is represented in fig. 24; their general appearance is not unlike a child's battledore, with its surface covered with spots. I have not been able satisfactorily to demonstrate its structure; but it appears io consist of two delicate tissues, having regular rows of conical spines on the upper one. As a test-object, these spots should be clearly and distinctly separated. When the light is thrown obliquely, they are blended together, appearing like a stripe of unequal breadth; similar to many of the other tests, it is the manner in which they are seen rather than the mere exhibition of them that should be observed. This object I employ for the same purpose as the leaf of an unknown species of moss belonging to the genus Hypnum, which, as it is difficult to procure, renders this substitute an acquisition to the microscopist.

Before I conclude this chapter, it may not be amiss to notice another class of objects, which by the vulgar are considered as positive proofs of the efficiency of an instrument; I allude to the animalcules. Nor does this opinion seem confined to those unacquainted with this subject; but we find it stated by Adams, in his quarto work on the Microscope, p. 4.30, that the Monas Termo (one of the most minute of all the animalcules very abundant in vegetable infusions), "eludes the power of the compound microscope, and is but imperfectly seen by the single." Now, all that is requisite for seeing this object, or any other of the same kind, is to cut off by stops, or otherwise, all superfluous light, so as to reduce the quantity and intensity $\ddagger$ of the illumination; for, when too much light

- The serratures on the surface of the human hair, especially those from an infant, afford excellent tests, and are very beautiful objects.

+ The form of these hairs varies in every species : in some they resemble the feather of the peacosk's tail in miniature; others are furnished with tufts of fine hair, and beset with spines.

$\ddagger$ The reader should observe that quantity and intensity are distinct from each other: thus, when we employ a small wax taper close to an object, it 


\section{Mr. Scrymgeour's Experiments to determine the Influence}

is admitted, these minute and delicate bodies are completely drowned. All that is necessary for seeing these objects, even in the ordinary compound microscopes (engiscopes), providing they have sufficient magnifying power, is to employ a faint illumination. If, however, the observer is desirous of examining the structure and organization of them, of course he must use an instrument of superior quality; for in this case not only sufficient magnifying power and proper illumination are necessary, but penetration and definition.

LVI. Narratize of Experiments made with the Seconds Pendulum, principally in order to determine the hitherto unassigned Amount of the Influence of certain minute Forces on its Rate of Motion. By Mr. James Scrumgeour.

[Continued from p. 251.]

MY next inquiry was to ascertain whether a pendulum of the same length made any difference in its time, accordingly as its weight was light or heavy. The only method of ascertaining this experimentally, is to employ the principle of convertibility adopted by Captain Kater in his experiments on the pendulum. The convertible pendulum which I employed was composed of two steel rods placed an inch separate from each other, and firmly joined by cross pieces at the ends as well as at intervals of their length. The knife-edges or portions of cylinders were fixed between the rods, at the points of oscillation and suspension.

In the following experiments the knife-edges were used, and the pendulum was adjusted, first with weights which, along with it, made the whole weight 8 pounds 10 ounces. When the heavy end of the pendulum was lowest, it vibrated exactly with the clock pendulum at an extent varying from $3^{\circ}$ to $2^{\circ} \cdot 5^{\circ}$; when the light end was lowest, at an extent of vibration varying from $2^{\circ}$ to $1^{\circ} 5$.

The pendulum was then transferred to the vessel in which the exhaustion was to be effected. When exhausted to a mean pressure of $1 \frac{1}{2}$ inch of mercury, the pendulum, with the light end lowest, vibrated with the clock pendulum at an extent of

will be intensely illuminated though the quantity of light is small; but if we employ the flame of a large lamp, \&c. at some distance from the object, its intensity will be small, though the quantity of light be great. It will be found generally preferable to employ a small quantity of intense light, rather than a larger portion of weak light; and, if possible, avoid the use of lenses or mirrors, either for condensing or changing the direction of the light. 


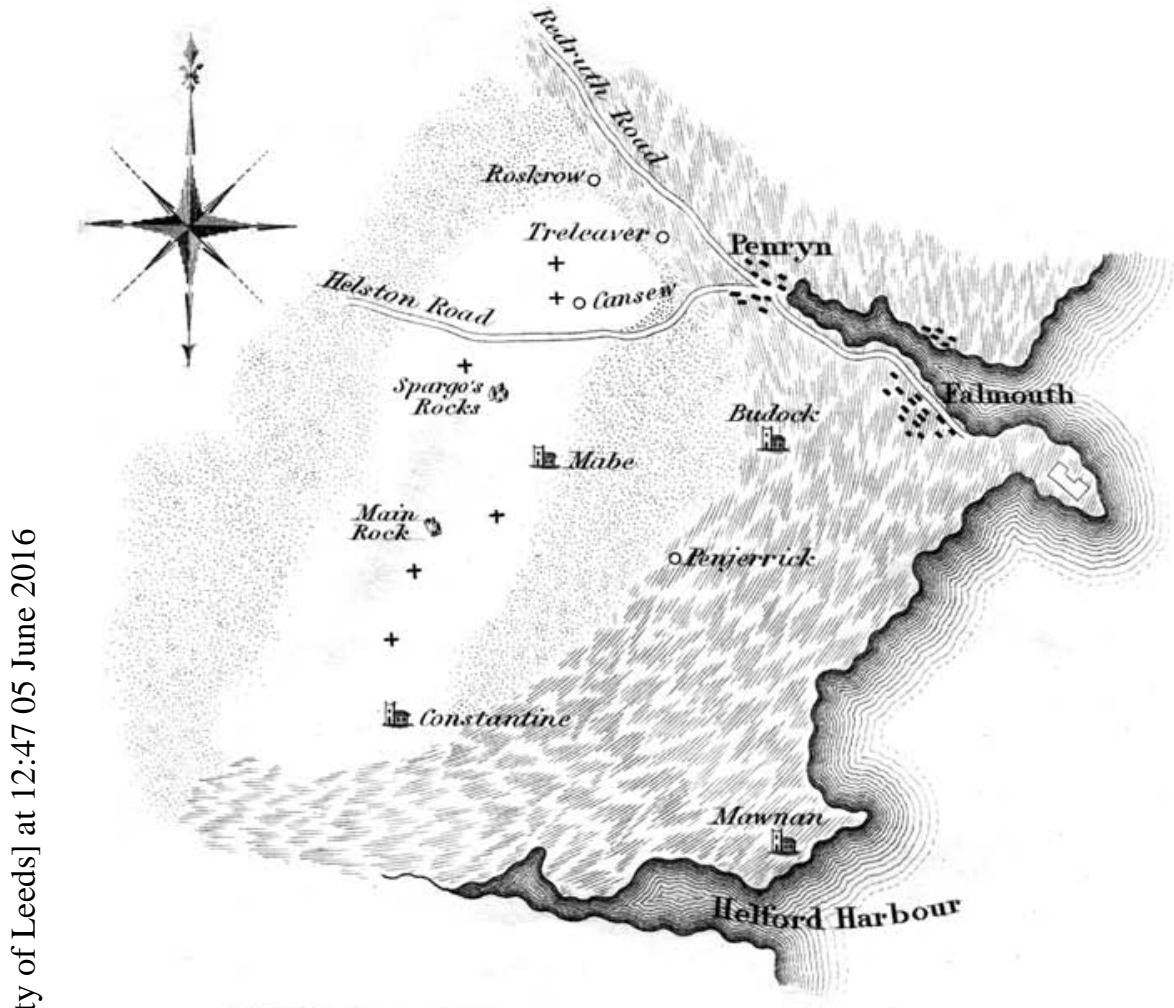

Mr.R.W. Fox's Map of the Granite District, near Penryn.
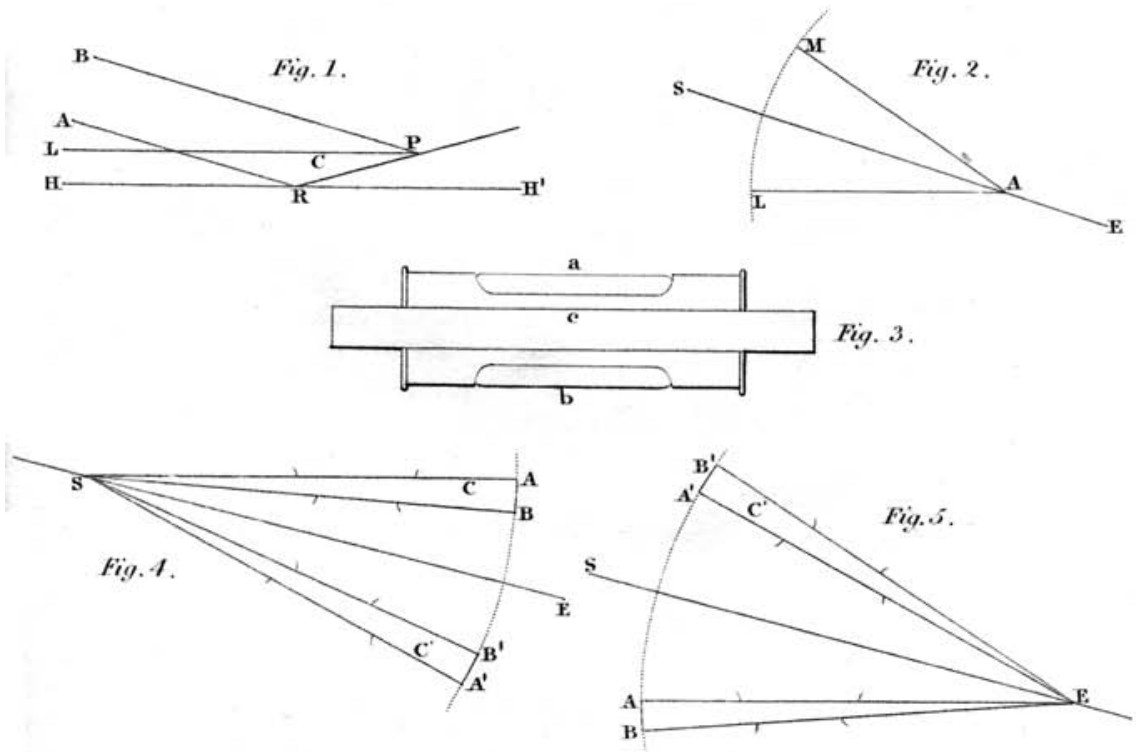

M!' Nixon on his Horizon Sector: 